\title{
Characterisation of fibre-rich powder and antioxidant capacity of Mangifera pajang $K$. fruit peels.
}

\begin{abstract}
The composition of bambangan peel dietary fibre (DF) and several properties related to its nutritional quality were investigated. The physicochemical properties and antioxidant capacity for the fibre-rich powder (FRP) were investigated, and the chemical composition for soluble dietary fibre (SDF) and insoluble dietary fibre (IDF) were also studied. The FRP had a high amount of total dietary fibre (TDF; $72.3 \mathrm{~g} / 100 \mathrm{~g}$ FRP) with a balanced SDF/IDF ratio $(46.3 / 53.7 \%)$. The FRP had a high glucose retardation index, water-holding capacity (WHC), oil-holding capacity (OHC), and swelling. The antioxidant capacity of the FRP, as determined by the DPPHradical dot assay using an Elisa reader, exhibited a strong potency due to the presence of associated total polyphenols $(98.3 \mathrm{mg} / \mathrm{g}$ FRP). These FRP characteristics indicated that bambangan peels are a rich source of DF, antioxidants, and other bioactive compounds that can be incorporated with food products to improve the nutraceutical properties of these products.
\end{abstract}

Keyword: Antioxidant capacity; Dietary fibre; Mangifera pajang peel; Physicochemical properties. 